\title{
Utility of Integrated Analysis of Pharmacogenomics and Pharmacometabolomics in Early Phase Clinical Trial: A Case Study of a New Molecular Entity
}

\author{
Jaeseong $\mathrm{Oh}^{1}$, Sojeong $\mathrm{Yi}^{2}$, Namyi $\mathrm{Gu}^{3}$, Dongseong Shin ${ }^{4}$, Kyung-Sang $\mathrm{Yu}^{1}$, \\ Seo Hyun Yoon ${ }^{1}$, Joo-Youn Cho ${ }^{1}$, In-Jin Jang ${ }^{1 *}$ \\ ${ }^{1}$ Department of Clinical Pharmacology and Therapeutics, Seoul National University College of Medicine and Hospital, Seoul, \\ 03080, Korea, ${ }^{2}$ Office of Clinical Pharmacology, Center for Drug Evaluation and Research, US Food and Drug Administration, \\ Silver Spring, MD 10903, USA, ${ }^{3}$ Department of Clinical Pharmacology and Therapeutics, Clinical Trial Center, \\ Dongguk University Ilsan Hospital, Dongguk University College of Medicine, Goyang 10326, Korea, ${ }^{4}$ Clinical Trials Center, \\ Gachon University Gil Medical Center, Incheon 21565, Korea
}

In this report, we present a case study of how pharmacogenomics and pharmacometabolomics can be useful to characterize safety and pharmacokinetic profiles in early phase new drug development clinical trials. During conducting a first-in-human trial for a new molecular entity, we were able to determine the mechanism of dichotomized variability in plasma drug concentrations, which appeared closely related to adverse drug reactions (ADRs) through integrated omics analysis. The pharmacogenomics screening was performed from whole blood samples using the Affymetrix DMET (Drug-Metabolizing Enzymes and Transporters) Plus microarray, and confirmation of genetic variants was performed using real-time polymerase chain reaction. Metabolomics profiling was performed from plasma samples using liquid chromatography coupled with quadrupole time-of-flight mass spectrometry. A GSTM1 null polymorphism was identified in pharmacogenomics test and the drug concentrations was higher in GSTM1 null subjects than GSTM1 functional subjects. The apparent drug clearance was 13-fold lower in GSTM1 null subjects than GSTM1 functional subjects ( $p<0.001$ ). By metabolomics analysis, we identified that the study drug was metabolized by cysteinylglycine conjugation in GSTM functional subjects but those not in GSTM1 null subjects. The incidence rate and the severity of ADRs were higher in the GSTM1 null subjects than the GSTM1 functional subjects. Through the integrated omics analysis, we could understand the mechanism of inter-individual variability in drug exposure and in adverse response. In conclusion, integrated multi-omics analysis can be useful for elucidating the various characteristics of new drug candidates in early phase clinical trials.

Keywords: clinical trial, metabolomics, new drug development, pharmacogenomics

\section{Introduction}

Improving efficiency is an important issue in new drug development. Although there have been considerable advances in science and technology, the number of approved new drugs per research spending has continuously declined over 60 years [1]. Dramatic improvements were made in pre-clinical research areas, but the successful clinical development rate of new drug candidates has not changed in recent years [1]. To improve efficiency in clinical trials, enrichment strategies were proposed in several disease areas, which enable precision medicine in drug development [2-4].

Various omics technologies have been used in recent clinical trials to elucidate the various characteristics of new drug candidates and to identify optimal subjects for the trials [5-10]. Pharmacogenomics is used in clinical trials to select appropriate patients and to further explain pharmacokinetics, pharmacodynamics, efficacy, and adverse drug reactions of new drug candidates [6-8]. Pharmacometabolomics can be used in clinical trials to identify drug targets, diagnosis disease, assess drug metabolic enzyme 
function in humans, and monitor the drug response and toxicity [9-13]. The importance of those evaluations is high in early phase clinical trials because it can help to simplify late phase clinical trials and increase the probability of success of clinical trials [5].

In this report, we present a case showing how pharmacogenomics and pharmacometabolomics were used in an early phase new drug development clinical trials. Drug X is a transient receptor potential vanilloid type 1 (TRPV1) antagonist class of new drug candidate that is under clinical development for the treatment of atopic dermatitis. In a first-in-human clinical trial for drug X, large inter-individual variability in the plasma concentration was observed after a single oral administration. Furthermore, moderate adverse drug reactions were observed in certain group of subjects. To perform subsequent clinical trials safely and efficiently, we needed to understand the cause of the inter-individual variability observed in the early phase clinical trial for drug X.

The aim of this study was to investigate the mechanism of the variability in plasma concentration of drug $\mathrm{X}$ and the adverse drug reaction by integrated multi-omics analysis using pharmacogenomics and pharmacometabolomics tools.

\section{Methods}

\section{Study samples}

The first-in-human clinical study enabled exploratory analysis of pharmacogenomics and pharmacometabolomic analysis. The study was approved by the Institutional Review Board of Seoul National University Hospital, Seoul, Korea
(H-1011-027-339) and was conducted in accordance with the principles of the Declaration of Helsinki and ICH Good Clinical Practice. All subjects provided written informed consent before any study-related procedure was performed.

Whole blood samples $(n=32)$ were obtained from every subject who received a single oral dose of drug $\mathrm{X}$ for the

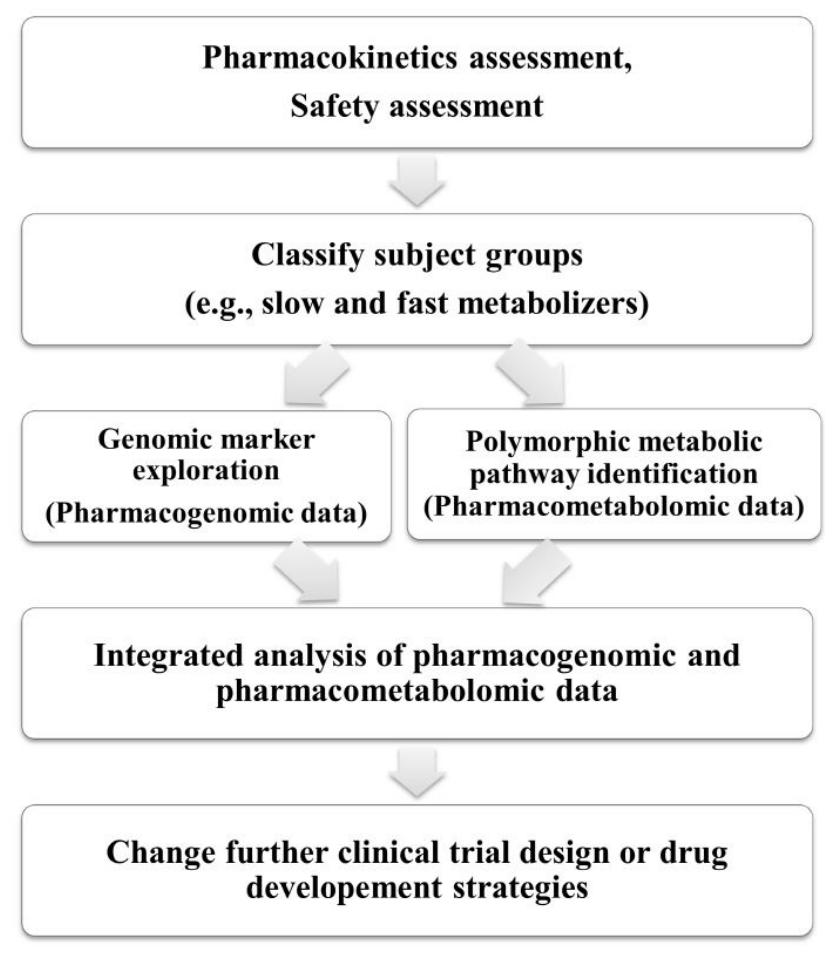

Fig. 2. Study flow.

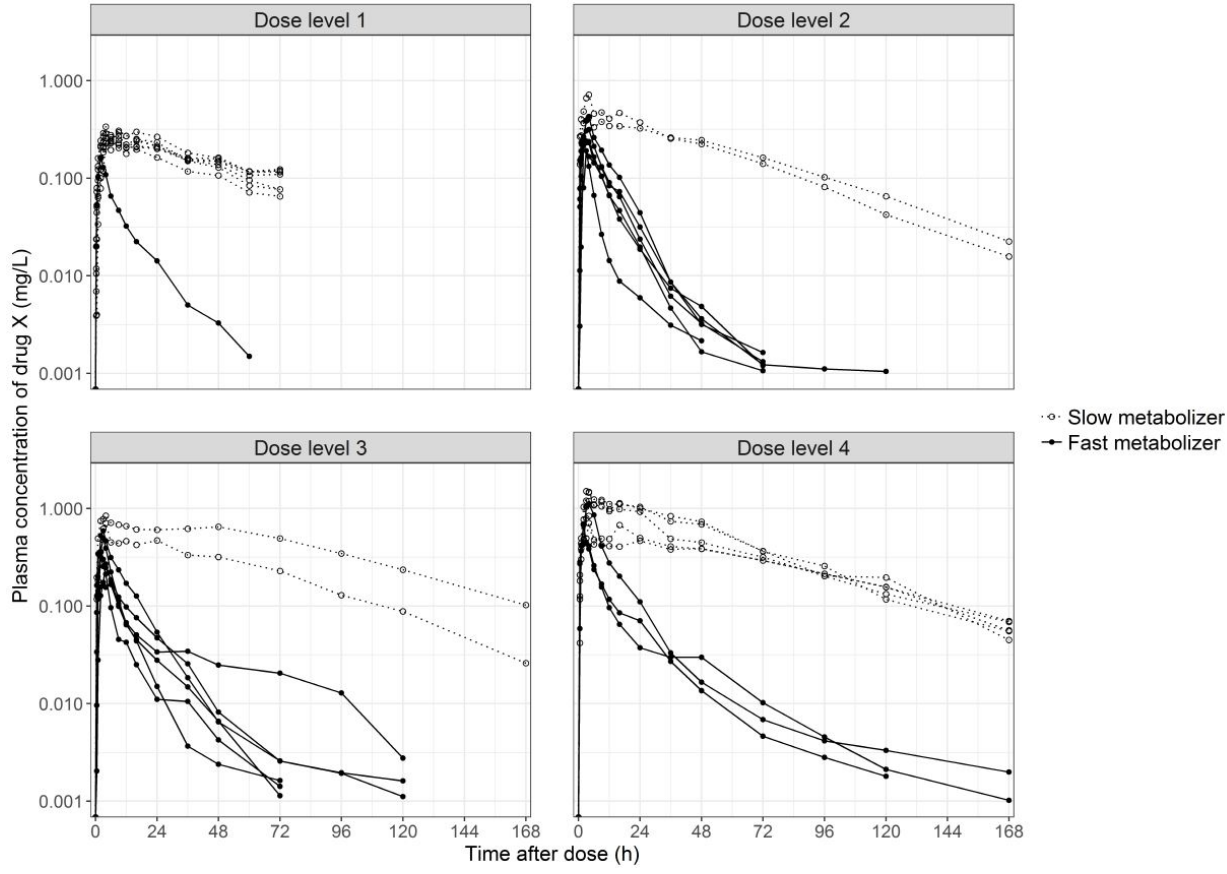

Fig. 1. Plasma concentration-time profiles of drug $\mathrm{X}$ after single oral administration. 
pharmacogenomic genotyping. Plasma samples $(n=8)$ were obtained from the subjects who received highest dose of drug $\mathrm{X}$ for the pharmacometabolomic analysis.

\section{Genotyping}

Genomic DNA was extracted from the whole blood using a QIAamp DNA Mini Kit (QIAgen, Hilden, Germany). The pharmacogenomics screening was performed using an Affymetrix DMET (Drug-Metabolizing Enzymes and Transporters) Plus microarray (Affymetrix, Santa Clara, CA, USA) at DNA Link Co. Ltd. (Seoul, Korea) according to the previously described method [14]. The microarray covered 1,931 single nucleotide polymorphisms (SNPs) and small insertions/deletions (indels) and 5 copy-number variations for 255 genes involved in the metabolism, transport, and excretion of drugs in humans and its utility and robustness has been validated in previous reports $[15,16]$. Furthemore, a real-time polymerase chain reaction (PCR) was performed using TaqMan SNP Genotyping Assays (Applied Biosystems, Foster City, CA, USA) to confirm the GSTM1 copy number variant identified from the microarray.

\section{Pharmacometabolomic analyses}

Metabolomic profiling to identify metabolites of drug X was performed in plasma samples using liquid chromatography (Agilent 1200 series, Agilent Technologies, Santa Clara, CA, USA) coupled with quadrupole timeof-flight mass spectrometry (Agilent 6530 Q-TOF MS, Agilent Technologies). To prepare the analyte, a $100 \mu \mathrm{L}$ of plasma sample was mixed with $400 \mu \mathrm{L}$ of acetonitrile. Five microliters of supernatant aliquot was injected onto the Agilent Zorbax C18 column (1.8 $\mu$ m particle size, $2.1 \times 50$ $\mathrm{mm}$; Agilent Technologies) at $30^{\circ} \mathrm{C}$ under gradient elution for $18 \mathrm{~min}$. The mobile phase consisted of a mixture of $0.1 \%$ formic acid in $2 \mathrm{mM}$ ammonium formate and $0.1 \%$ formic acid in acetonitrile. Positive ion electrospray ionization mode was used for mass spectrometry. The phase I and phase II metabolites of drug X were identified by molecular feature extraction of Agilent MassHunter Qualitative Software version B.03.01 (Agilent Technologies).

\section{Data analysis}

The pharmacokinetic parameters of drug X were analyzed by noncompartmental analysis and presented using descriptive statistics. The maximum plasma concentration $\left(\mathrm{C}_{\max }\right)$ and the area under the plasma concentration-time curve from 0 to the last measurable concentration (AUClast), which represents the systemic exposure to drug $\mathrm{x}$, is summarized by genotype and dose groups. The apparent clearance $(\mathrm{CL} / \mathrm{F})$, which represents the capacity of drug elimination from the body, was pooled among the dose groups, and the Mann-Whitney U test was used to compare the $\mathrm{CL} / \mathrm{F}$ between the genotype groups. A p-value of $<0.05$ was considered to be statistically significant. The number of drug-related adverse events (AEs) between the genotype groups was compared using the chi-square test and Fisher's exact test. Statistical analyses were performed using SAS software version 9.3 (SAS Institute Inc., Cary, NC, USA).

\section{Results}

\section{Subject classification and study flow}

After pharmacokinetics and safety assessment of the original first-in-human clinical study, the subjects were clearly classified into two groups, namely, slow and fast metabolizers (Fig. 1). After the classification, genomic marker exploration was performed by pharmacogenomic analysis and metabolic pathway identification was

Table 1. Pharmacokinetic parameters of drug $X$ following single oral dose

\begin{tabular}{|c|c|c|c|c|c|c|c|c|c|c|c|}
\hline \multirow[b]{2}{*}{ Parameters } & \multicolumn{2}{|c|}{ Dose level 1} & \multicolumn{2}{|c|}{ Dose level 2} & \multicolumn{2}{|c|}{ Dose level 3} & \multicolumn{2}{|c|}{ Dose level 4} & \multicolumn{3}{|c|}{ Total } \\
\hline & $\underset{(-/-)}{\operatorname{GSTM1}}$ & $\begin{array}{c}\text { GSTM1 } \\
(-/+) \\
\text { or } \\
(+/+)\end{array}$ & $\begin{array}{c}\text { GSTM1 } \\
(-/-)\end{array}$ & $\begin{array}{c}\text { GSTM1 } \\
(-/+) \\
\text { or } \\
(+/+)\end{array}$ & $\begin{array}{c}\text { GSTM1 } \\
(+/-)\end{array}$ & $\begin{array}{c}\text { GSTM1 } \\
(-/+) \\
\text { or } \\
(+/+)\end{array}$ & $\underset{(-/-)}{\text { GSTM1 }}$ & $\begin{array}{c}\text { GSTM1 } \\
(-/+) \\
\text { or } \\
(+/+)\end{array}$ & $\underset{(-/-)}{\operatorname{GSTM1}}$ & $\begin{array}{c}\text { GSTM1 } \\
(-/+) \\
\text { or } \\
(+/+)\end{array}$ & $\begin{array}{c}\text { p- } \\
\text { value }\end{array}$ \\
\hline No. & 7 & 1 & 2 & 6 & 2 & 6 & 5 & 3 & 16 & 16 & - \\
\hline $\mathrm{CL} / \mathrm{F}(\mathrm{L} / \mathrm{h})$ & $1.1 \pm 0.3$ & 14.6 & $1.8 \pm 0.0$ & $21.6 \pm 12.2$ & $2.1 \pm 1.0$ & $30.6 \pm 11.5$ & $3.3 \pm 0.8$ & $30.1 \pm 10.8$ & $2.0 \pm 1.1$ & $26.1 \pm 11.7$ & $<0.001$ \\
\hline $\mathrm{C}_{\max }(\mathrm{mg} / \mathrm{L})$ & $0.27 \pm 0.04$ & 0.16 & $0.57 \pm 0.21$ & $0.28 \pm 0.09$ & $0.78 \pm 0.09$ & $0.37 \pm 0.15$ & $1.14 \pm 0.36$ & $0.74 \pm 0.35$ & - & - & - \\
\hline $\begin{array}{l}\mathrm{AUC}_{\text {last }} \\
(\mathrm{mg} \cdot \mathrm{h} / \mathrm{L})\end{array}$ & $11.87 \pm 1.47$ & 1.35 & $26.85 \pm 0.39$ & $2.73 \pm 1.08$ & $51.82 \pm 23.63$ & $3.61 \pm 1.33$ & $59.72 \pm 14.10$ & $7.33 \pm 3.26$ & - & - & - \\
\hline$t_{1 / 2}(h)$ & $47.0 \pm 12.7$ & 13.4 & $32.0 \pm 0.0$ & $69.6 \pm 28.5$ & $35.0 \pm 7.8$ & $58.6 \pm 28.0$ & $38.9 \pm 6.9$ & $51.2 \pm 13.8$ & - & - & - \\
\hline
\end{tabular}

Values are presented as mean \pm standard deviation.

GSTM1(-/), subject with glutathione-S-transferase M1 null function; GSTM1(-/+) or $(+/+)$, subject with one or two glutathione-S-transferase M1 functional allele; $C L / F$, apparent clearance; $C_{\max }$, maximum plasma concentration; $A \cup C_{\text {last, }}$ area under the plasma concentration-time curve from 0 to the last measurable concentration; $t_{1 / 2}$, terminal elimination half-life. 
performed by drug pharmacometabolomic analysis. The genomic marker data was integrated with metabolic pathway data to understand the mechanism of the inter-individual variability. The overall study flow is presented in Fig. 2.

\section{Pharmacogenomics analysis results}

In the slow metabolizer subjects, homozygous deletion of the glutathione-S-transferase M1 gene (GSTM1 null), which leads to loss of enzyme function, was identified from the DMET Plus microarray. The systemic exposure to drug X,

(A)

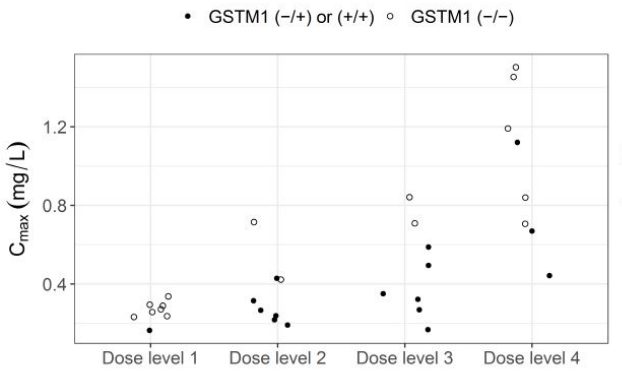

(C)

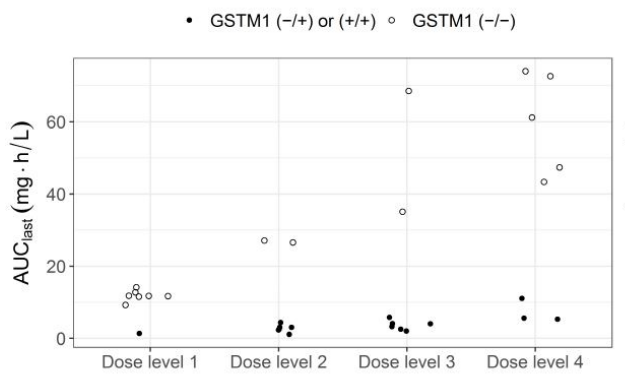

(A)

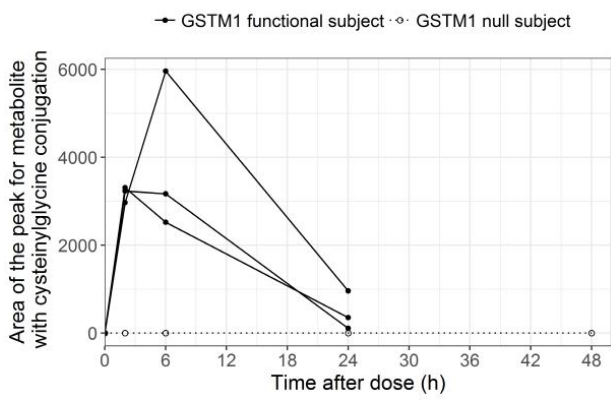

(C)

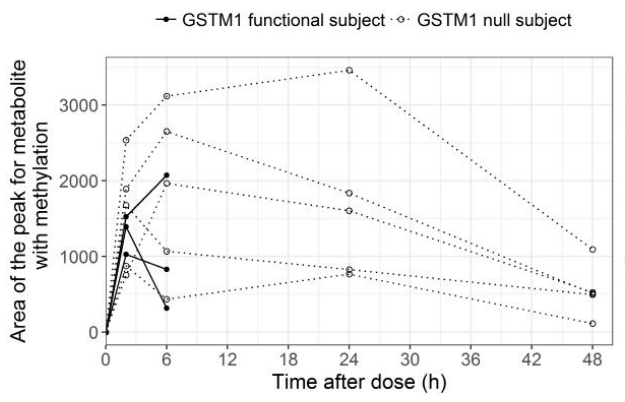

(B)

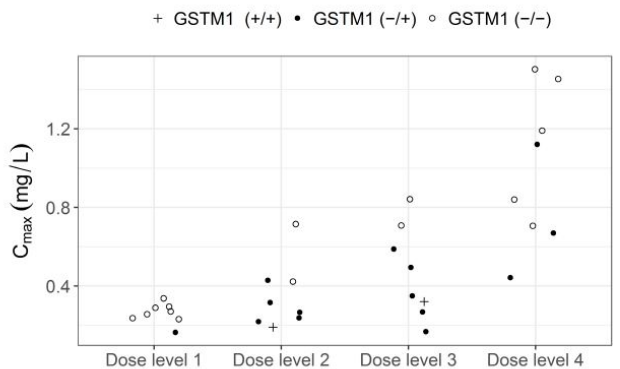

(D)

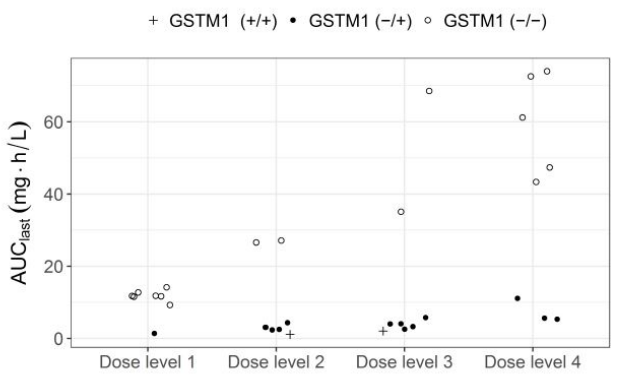

(B)

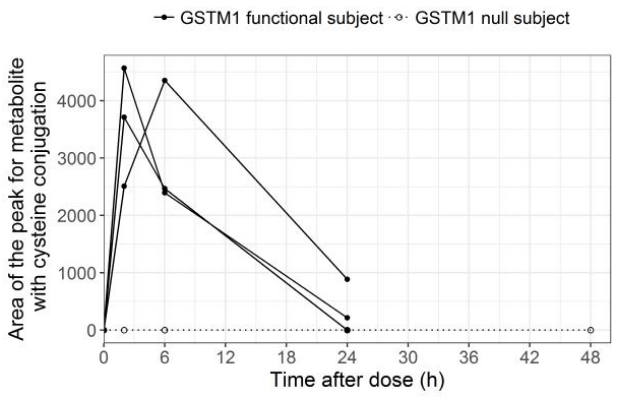

(D)

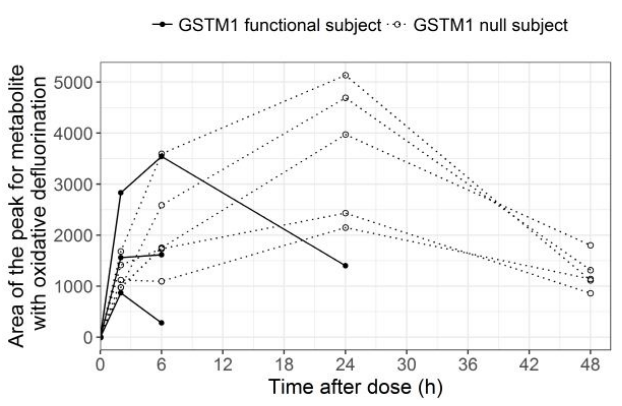

Fig. 3. (A-D) Comparison of the pharmacokinetic parameters of drug $X$ among the different CSTM1 genotype groups. GSTM1(-I-), subject with glutathione-S-transferase M1 null function; GSTM1(-/+), subject with one glutathione-S-transferase M1 functional allele; $\operatorname{GSTM} 1(+/+)$, subject with two glutathione-S-transferase $\mathrm{M} 1$ functional allele; $\mathrm{C}_{\max }$ maximum plasma concentration; $A \cup C_{\text {last, }}$ area under the plasma concentration-time curve from 0 to the last measurable concentration.

Fig. 4. (A-D) Plasma drug $X$ metabolite-time profiles after single oral administration. 


\section{Pharmacometabolomic analyses result}

A total of four metabolites of drug X (cysteine conjugation, cysteinylglycine conjugation, methylation, oxidative defluorination) were observed from the plasma samples. Among these metabolites, cysteine and cysteinylglycine conjugation metabolites were detected in functional GSTM1 subjects, but they were absent in GSTM1 null subjects (Fig. 4). The areas under the peak for methylation and oxidative defluorination metabolites were higher in the GSTM1 null subjects than the functional GSTM1 subjects (Fig. 4).

\section{GSTM1 null genotype and drug-related AE}

The number of drug-related AEs and the number of subjects with drug-related AEs were higher in the GSTM1 null subjects than the GSTM1 functional subjects, although it failed to reach statistical significance (Table 2). Furthermore, the AEs were more severe in the GSTM1 null subjects than the GSTM1 functional subjects. Three subjects in the GSTM1 null group experienced AEs with moderate severity, whereas none in the GSTM1 functional group experienced those AEs. Those AEs were feeling hot, feeling cold and a burning sensation, and these sensations are frequently reported AEs in TRPV1 antagonist class drugs $[17,18]$.

\section{Discussion}

Through the integrated omics analysis, we could understand the reason for the inter-individual pharmacokinetic variability observed in the first-in-human trial for drug X. The GSTs are a group of phase II enzymes that play an important role in the biotransformation of various xenobiotic and endogenous compounds by conjugation with glutathione [19]. GSTM1 is a $\mu$ class isoform of GST, and the GSTM1 gene is highly polymorphic in humans. Homozygous deletion of the GSTM1 gene is observed in various ethnic groups, and its frequency ranged from $29.6 \%$ to $56.2 \%$ [20, 21]. The frequency of GSTM1 null subjects was $50 \%$ in this study, consistent with the previously reported GSTM1 null frequency in Koreans [20]. The loss of catalytic function is suggested to be reason for decreased systemic clearance of drug $\mathrm{X}$ in GSTM1 null subjects. This hypothesis was supported by the metabolite analysis showing that no cysteine conjugation or cysteinylglycine conjugation metabolites of drug X were observed in GSTM1 null subjects.

The effect of the GSTM1 null polymorphism on systemic exposure to drug $\mathrm{X}$ was reproduced in subsequent clinical trials. The GSTM1 null subjects showed higher plasma concentration and lower $\mathrm{CL} / \mathrm{F}$ of drug $\mathrm{X}$ than functional

Table 2. Summary of drug-related AEs

\begin{tabular}{lccc}
\hline & $\begin{array}{c}\text { GSTM1 null subject } \\
(\mathbf{n}=\mathbf{1 6})\end{array}$ & $\begin{array}{c}\text { GSTM1 functional subject } \\
(\mathbf{n}=\mathbf{1 6})\end{array}$ & p-value \\
\hline No. of drug-related AEs & 26 & 16 & $0.123^{\mathrm{a}}$ \\
No. of subjects with drug-related AEs & $9(56.3)$ & $6(37.5)$ & $0.288^{\mathrm{a}}$ \\
No. of subjects with mild AEs & $8(50)$ & $6(37.5)$ & $0.476^{\mathrm{a}}$ \\
No. of subjects with moderate AEs & $3(18.8)$ & 0 & $0.226^{\mathrm{b}}$ \\
No. of subjects with severe AEs & $0(0)$ & - \\
\hline
\end{tabular}

Values are presented as number (\%).

$\mathrm{AE}$, adverse event.

${ }^{\mathrm{a}}$ Chi-squared test; ${ }^{\mathrm{b}}$ Fisher's exact test.

Table 3. Pharmacokinetic parameters of drug $X$ following multiple oral doses

\begin{tabular}{|c|c|c|c|c|c|c|c|}
\hline \multirow[b]{2}{*}{ Parameters } & \multicolumn{2}{|c|}{ Dose level 5} & \multicolumn{2}{|c|}{ Dose level 6} & \multicolumn{3}{|c|}{ Total } \\
\hline & $\begin{array}{c}\text { GSTM1 } \\
(-/-)\end{array}$ & $\begin{array}{c}\text { GSTM1 } \\
(-/+)\end{array}$ & $\begin{array}{c}\text { GSTM1 } \\
(-/-)\end{array}$ & $\begin{array}{c}\text { GSTM1 } \\
(-/+)\end{array}$ & $\begin{array}{c}\text { GSTM1 } \\
(-/-)\end{array}$ & $\begin{array}{c}\text { GSTM1 } \\
(-/+)\end{array}$ & p-value \\
\hline No. & 7 & 1 & 5 & 3 & 12 & 4 & \\
\hline $\mathrm{CL} / \mathrm{F}_{\mathrm{ss}}(\mathrm{L} / \mathrm{h})$ & $0.4 \pm 0.1$ & 10.4 & $0.4 \pm 0.1$ & $7.5 \pm 4.6$ & $0.4 \pm 0.1$ & $8.2 \pm 4.0$ & 0.001 \\
\hline $\mathrm{C}_{\max , \mathrm{ss}}(\mathrm{mg} / \mathrm{L})$ & $0.23 \pm 0.03$ & 0.06 & $0.47 \pm 0.14$ & $0.15 \pm 0.04$ & - & - & - \\
\hline $\mathrm{AUC}_{\tau, \mathrm{ss}}(\mathrm{mg} \cdot \mathrm{h} / \mathrm{L})$ & $4.25 \pm 0.54$ & 0.41 & $8.26 \pm 1.85$ & $1.22 \pm 0.52$ & - & - & - \\
\hline $\mathrm{t}_{1 / 2, \mathrm{ss}}(\mathrm{h})$ & $36.3 \pm 4.7$ & 42.0 & $37.2 \pm 3.1$ & $72.3 \pm 3.4$ & - & - & - \\
\hline
\end{tabular}

Values are presented as mean \pm standard deviation.

GSTM1(-/-), subject with glutathione-S-transferase M1 null function; GSTM1(-/+), subject with one glutathione-S-transferase M1 functional allele; $C L / F_{s s}$, apparent clearance at steady state; $C_{m a x, s s}$ maximum plasma concentration at steady state; $A \cup C \tau, s s$, area under the plasma concentration-time curve within a dosing interval at steady state; $t_{1 / 2, s,}$, terminal elimination half-life at steady state. 


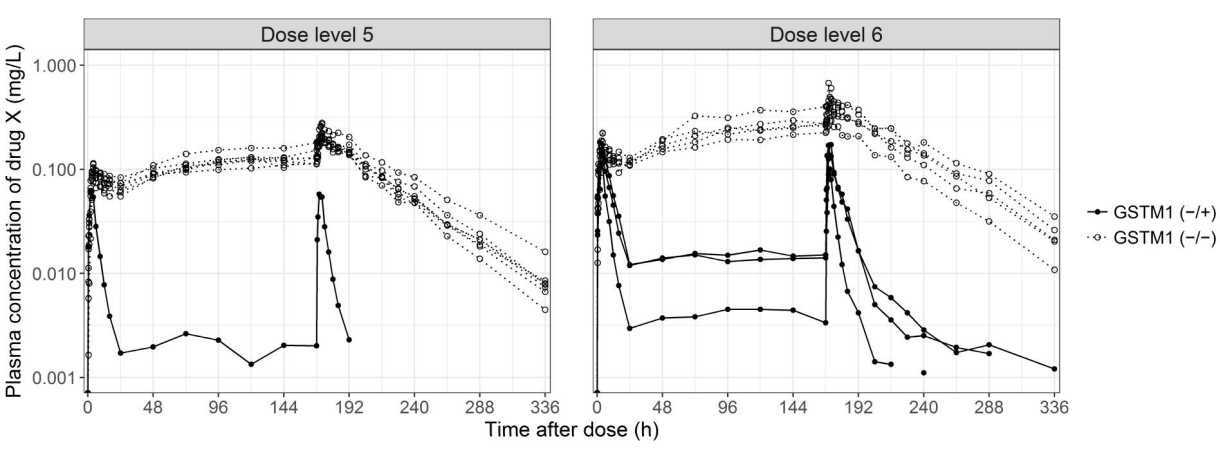

Fig. 5. Plasma concentration-time profiles of drug $\mathrm{X}$ after multiple oral administration. CSTM1(-/), subject with glutathione-S-transferase M1 null function; GSTM1 $(-/+)$, subject with one glutathione-S-transferase M1 functional allele.
GSTM1 subjects after multiple oral administrations (Table 3, Fig. 5). The clinical development strategy for drug X needed to be modified to reflect these results because the GSTM1 null variant was a major determining factor for drug exposure, as well as drug-related AEs, and because the frequency of GSTM1 nulls is approximately half of total population. A personalized drug development strategy applying different oral doses based on the GSTM1 genotype or switching to a different formulation with limited systemic exposure was needed for safe and efficient development of drug $\mathrm{X}$. drug $\mathrm{X}$ is currently in clinical development as a topical formulation to limit systemic absorption.

Integrated multi-omics analysis can be a useful tool to evaluate the diverse characteristics of new drug candidates in clinical situations. The importance of integration of multi-omics data is growing in many research areas, including pharmaceutical research and development [22-24]. As shown in this case study, integrated multi-omics analysis can be used to explain the cause of inter-individual variability in drug exposure and adverse drug reactions observed in clinical development. This information can be important when making decisions for further drug development strategy.

In conclusion, integrated multi-omics analysis can be useful in early phase clinical trials for elucidating the various characteristics of new drug candidates that could not be produced through traditional clinical trial methods.

ORCID: Jaeseong Oh: https://orcid.org/0000-0001-62758587; Sojeong Yi: https://orcid.org/0000-0001-6659-1585; Namyi Gu: https://orcid.org/0000-0002-0182-0532; Dongseong Shin: https://orcid.org/0000-0002-4227-374 X; Kyung-Sang Yu: https://orcid.org/0000-0003-0921-722 5; Seo Hyun Yoon: https://orcid.org/0000-0002-2163-279 0; Joo-Youn Cho: https://orcid.org/0000-0001-9270-8273; In-Jin Jang: https://orcid.org/0000-0002-8384-3139

\section{Authors' contribution}

\section{Conceptualization: SY, K-SY, I-JJ}

Data curation: SY, NG, DS

Formal analysis: SY, JO

Funding acquisition: I-JJ

Methodology: SHY, J-YC

Writing - original draft: JO, SHY

Writing - review \& editing: SY, NG, DS, K-SY, J-YC, I-JJ

\section{Acknowledgments}

This research was supported by a grant of the Korea Health Technology R\&D Project through the Korea Health Industry Development Institute (KHIDI), funded by the Ministry of Health \& Welfare, Republic of Korea (grant number : HI15C1543).

\section{References}

1. Scannell JW, Blanckley A, Boldon H, Warrington B. Diagnosing the decline in pharmaceutical R\&D efficiency. Nat Rev Drug Discov 2012;11:191-200.

2. Merlo-Pich E, Alexander RC, Fava M, Gomeni R. A new population-enrichment strategy to improve efficiency of placebo-controlled clinical trials of antidepressant drugs. Clin Pharmacol Ther 2010;88:634-642.

3. Freidlin B, Korn EL. Biomarker enrichment strategies: matching trial design to biomarker credentials. Nat Rev Clin Oncol 2014;11:81-90.

4. Hollingsworth SJ. Precision medicine in oncology drug development: a pharma perspective. Drug Discov Today 2015;20: 1455-1463.

5. Roses AD. Pharmacogenetics and drug development: the path to safer and more effective drugs. Nat Rev Genet 2004;5:645656.

6. Ahn C. Pharmacogenomics in drug discovery and development. Genomics Inform 2007;5:41-45.

7. Harper AR, Topol EJ. Pharmacogenomics in clinical practice and drug development. Nat Biotechnol 2012;30:1117-1124.

8. Liou SY, Stringer F, Hirayama M. The impact of pharmacogenomics research on drug development. Drug Metab Pharmacokinet 2012;27:2-8.

9. Wishart DS. Emerging applications of metabolomics in drug discovery and precision medicine. Nat Rev Drug Discov 2016; 
$15: 473-484$

10. Robertson DG, Frevert U. Metabolomics in drug discovery and development. Clin Pharmacol Ther 2013;94:559-561.

11. Kaddurah-Daouk R, Weinshilboum RM; Pharmacometabolomics Research Network. Pharmacometabolomics: implications for clinical pharmacology and systems pharmacology. Clin Pharmacol Ther 2014;95:154-167.

12. Shin KH, Choi MH, Lim KS, Yu KS, Jang IJ, Cho JY. Evaluation of endogenous metabolic markers of hepatic CYP3A activity using metabolic profiling and midazolam clearance. Clin Pharmacol Ther 2013;94:601-609.

13. Kim B, Lee J, Shin KH, Lee S, Yu KS, Jang IJ, et al. Identification of omega- or (omega-1)-hydroxylated medium-chain acylcarnitines as novel urinary biomarkers for CYP3A activity. Clin Pharmacol Ther 2018;103:879-887.

14. Yi S, An H, Lee H, Lee S, Ieiri I, Lee Y, et al. Korean, Japanese, and Chinese populations featured similar genes encoding drug-metabolizing enzymes and transporters: a DMET Plus microarray assessment. Pharmacogenet Genomics 2014;24: 477-485.

15. Fernandez CA, Smith C, Yang W, Lorier R, Crews KR, Kornegay $\mathrm{N}$, et al. Concordance of DMET plus genotyping results with those of orthogonal genotyping methods. Clin Pharmacol Ther 2012;92:360-365.

16. He YJ, Misher AD, Irvin W Jr, Motsinger-Reif A, McLeod HL, Hoskins JM. Assessing the utility of whole genome amplified DNA as a template for DMET Plus array. Clin Chem Lab Med 2012;50:1329-1334.

17. Rowbotham MC, Nothaft W, Duan WR, Wang Y, Faltynek C,
McGaraughty S, et al. Oral and cutaneous thermosensory profile of selective TRPV1 inhibition by ABT-102 in a randomized healthy volunteer trial. Pain 2011;152:1192-1200.

18. Quiding H, Jonzon B, Svensson O, Webster L, Reimfelt A, Karin A, et al. TRPV1 antagonistic analgesic effect: a randomized study of AZD1386 in pain after third molar extraction. Pain 2013;154:808-812.

19. Salinas AE, Wong MG. Glutathione S-transferases: a review. Curr Med Chem 1999;6:279-309.

20. Kurose K, Sugiyama E, Saito Y. Population differences in major functional polymorphisms of pharmacokinetics/pharmacodynamics-related genes in Eastern Asians and Europeans: implications in the clinical trials for novel drug development. Drug Metab Pharmacokinet 2012;27:9-54.

21. Kasthurinaidu SP, Ramasamy T, Ayyavoo J, Dave DK, Adroja DA. GST M1-T1 null allele frequency patterns in geographically assorted human populations: a phylogenetic approach. PLoS One 2015;10:e0118660.

22. Fondi M, Lio P. Multi-omics and metabolic modelling pipelines: challenges and tools for systems microbiology. Microbiol Res 2015;171:52-64.

23. Kamoun A, Idbaih A, Dehais C, Elarouci N, Carpentier C, Letouze $\mathrm{E}$, et al. Integrated multi-omics analysis of oligodendroglial tumours identifies three subgroups of $1 \mathrm{p} / 19 \mathrm{q}$ co-deleted gliomas. Nat Commun 2016;7:11263.

24. Schumacher A, Rujan T, Hoefkens J. A collaborative approach to develop a multi-omics data analytics platform for translational research. Appl Transl Genom 2014;3:105-108. 